\title{
Edge Oriented Spatial Image Filtering Technique
}

\author{
N.Ramakrishna \\ Assistant Professor, \\ ETM Department, \\ GNITS, Hyderabad-08.
}

\author{
Dr.V.S.Mallela \\ Professor, \\ EEE Department, \\ GNITS, Hyderabad-08.
}

\begin{abstract}
Many techniques have been reported earlier in open literature in the area of digital image enhancement. In this paper, a novel approach is presented which improves the accuracy and minimizes the blurring effect with edge preservation. The work involved construction of a new filter using weighted average method, an edge filter and a combiner. The new filter thus constructed has been tested and evaluated for its performance with several test images. The results are encouraging in terms of de-noising and edge preservation. This technique may find practical applications in the area of preprocessing of digital images.
\end{abstract}

\section{Keywords}

Image enhancement, weighted Average filter, edge filter

\section{INTRODUCTION}

The principal objective of image enhancement [4, 5, and 6] is to modify attributes (input gray level) of an image to make it more suitable for a given task and a specific observer. In this paper only spatial domain is considered. During this process, one or more attributes of the image are modified. The choice of attributes and the way they are modified are specific to a given task. Moreover, observer-specific factors, such as the human visual system and the observer's experience, will introduce a great deal of subjectivity into the choice of image enhancement methods. There exist many techniques that can enhance a digital image without spoiling it. Image enhancement simply means, transforming an image, say, $\mathrm{f}$ into an image $\mathrm{g}$ using a transformation, $\mathrm{T}$ [1][7]. The values of pixels in images $\mathrm{f}$ and $\mathrm{g}$ are denoted by $\mathrm{I}$ and $\mathrm{O}$, respectively. The pixel values $\mathrm{I}$ and $\mathrm{O}$ are related by the fundamental relationship

$\mathrm{O}=\mathrm{T}(\mathrm{I})$

The results of this transformation are mapped into the gray scale in the range $[0, \mathrm{~L}-1]$, where $\mathrm{L}=2 \mathrm{k}, \mathrm{k}$ being the number of bits; for an 8-bit image the range of pixel values will be [0, 255]. The block diagram of the proposed new filter and its details are presented in section 2 . The methodology used for construction of the new filter is explained in section 3 . The new filter is tested with several test images and the results are presented in section 4 along with a discussion. The paper concludes with the advantages of digital image enhancement with edge preservation in section 4.

\section{BLOCK DIAGRAM OF PROPOSED NEW FILTER}

The filtering scheme that removes uniform noise with edge preservation properties is shown in the figure 2.1. A weighted Average Filter reduces the noise by blurring the image. On the other hand an Edge filter detects the high frequency content of an image i.e. the image edges. Both images are fed to combiner. The combiner output is the de-noised image.

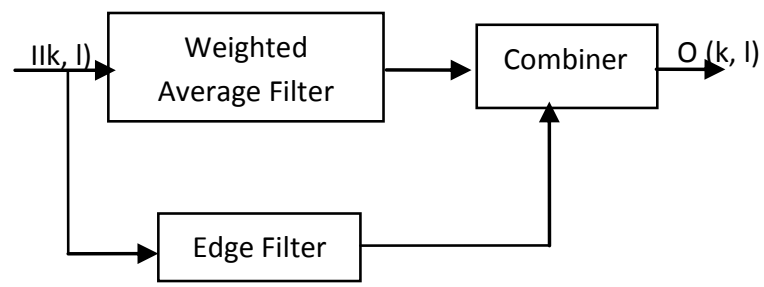

Fig2.1: Block Diagram of proposed new Filter.

An Image corrupted by Gaussian noise is the fed to weighted Average Filter. The edge filter is a non-linear High pass Filter. This Non-Linear High pass Filter is configured to act like an edge detector and preserves all the edges in four different arrays; vertical, horizontal, left diagonal and right diagonal. The Combiner Algorithm combines the weighted Average Filter Output (blurred) and the High Frequency content (edges) of the Image. Let $x$ and $I$ represent a two dimensional sequence, a noise free image and a noisy image (Gaussian noise) of $p$ rows and $q$ columns.

$$
I(i, j)=x(i, j)+\mathfrak{y}(i, j)
$$

Where $i=1,2 \ldots p, j=1,2 \ldots q$ and $n(i, j)$ is the $(i, j)$ th sample of the Gaussian noise sequence, $n$.

\subsection{Models of Filters:}

\subsubsection{Weighted Average Filter}

The average filtering [4, 5, and 6] is the most preferred technique for cleaning the signals confounded by Gaussian noise. A point window mask of size $(2 \mathrm{~N}+1) \times(2 \mathrm{~N}+1)$, representing an odd size is made to slide over the noisy input image for calculating average of samples.

The average of the samples inside the window mask is used as the filter output at each point and associated with the center sample inside the window. The novel approach adopted here is assigning the centre point the highest weight and then reducing the value of the coefficients as a function of increasing distance from the origin (centre point). This is an implicit attempt to 
reduce blurring in the smoothing process. The general implementation for filtering a $\mathrm{p} \times \mathrm{q}$ image with a weighted average filter is given by

$$
\begin{gathered}
\mathrm{ma}(\mathrm{x}, \mathrm{y})=\frac{\sum_{\mathrm{s}=-\mathrm{a}}^{\mathrm{a}} \sum_{\mathrm{t}=-\mathrm{b}}^{\mathrm{b}} \mathrm{w}(\mathrm{s}, \mathrm{t}) \mathrm{f}(\mathrm{x}+\mathrm{s}, \mathrm{y}+\mathrm{t})}{\sum_{\mathrm{s}=-\mathrm{a}}^{\mathrm{a}} \sum_{\mathrm{t}=-\mathrm{b}}^{\mathrm{b}} \mathrm{w}(\mathrm{s}, \mathrm{t})} \\
\mathrm{x}=0,1,2, \ldots \mathrm{M}-1, \mathrm{y}=0,1,2 \ldots \mathrm{N}-1
\end{gathered}
$$

\subsubsection{Edge Filter}

Edge filter [4][2] algorithm for one-dimensional signals discards the samples by comparing the absolute value of the difference in amplitude between two samples with a pre-selected threshold value. This algorithm is modified to detect and preserve horizontal, vertical, left diagonal and right diagonal edges of an image. The high pass filter algorithm separates image edges by sliding a space-ordered window of size $3 \times 3$ over the noisy input image, y. The filter algorithm used is given by $f(x, y)$ where

$$
f(x, y)=\left[\begin{array}{ccc}
y(k-1, l-1) & y(k-1, l) & y(k-1, l+1) \\
y(k, l-1) & y(k, l) & y(k, l+1) \\
y(k+1, l-1) & y(k+1, l) & y(k+1, l+1)
\end{array}\right] .
$$

The centre sample $\mathrm{y}(\mathrm{k}, \mathrm{l})$ is detected as a horizontal edge If $|\mathrm{y}(\mathrm{k}, \mathrm{l}-1)-\mathrm{y}(\mathrm{k}, \mathrm{l})| \geq \Delta \mathrm{t}$ OR $|\mathrm{y}(\mathrm{k}, \mathrm{l})-\mathrm{y}(\mathrm{k}, \mathrm{l}+1)| \geq \Delta \mathrm{t}, \Delta \mathrm{t}$ is the user defined threshold value and it is preserved in the $(\mathrm{k}, \mathrm{l})^{\mathrm{th}}$ location of a two dimensional array $\{\mathrm{h} 1\}$.Similarly, $\mathrm{y}(\mathrm{k}, \mathrm{l})$ is recognized as a vertical edge, as a left diagonal edge and a right diagonal edge, and is preserved in an array $\{\mathrm{h} 2\},\{\mathrm{h} 3\}$ and $\mathrm{h} 4$ respectively.

\subsubsection{Combiner}

The weighted average filter averages the image pixels at each point for reducing Gaussian noise. In this process, the image edges and their neighbourhood are blurred. The number of samples blurred about and including an edge sample along any direction (horizontal, vertical, diagonal) are $2 \mathrm{~N}+1$. The equations used for the combiner filter are

$$
=\left[\begin{array}{cc}
m a(i, j), k=i, l=j & \text { if } h a(i, j)=0, a=1,2,3,4) \\
y(i, j), k=i, l=j-N+d & \text { if } h 1(i, j)) \neq 0 ; \\
d=0,1,2 \ldots \ldots .2 N & \\
y(i, j), k=i-N+d, l=j & \text { if } h 2(i, j)) \neq 0 ; \\
d=0,1,2 \ldots \ldots .2 N & \\
y(i, j), k=i-N+d, l=j-N+d & \text { if } h 3(i, j)) \neq 0 ; \\
d=0,1,2 \ldots \ldots .2 N & \\
y(i, j), k=i-N+d, l=j+N-d & \text { if } h 4(i, j)) \neq 0 ;
\end{array}\right.
$$

Where $i=1,2 \ldots p$ and $j=1,2 \ldots q$.

If $y(k, 1)$ does not satisfy the criteria for an edge horizontally, vertically or diagonally, then it is declared a non-edge component and therefore, $(k, 1)$ th location of $\{h a\}, a=1,2,3$ and 4 is stored with zero. Thus, the high pass filter detects, separates and preserves the image edges.

\section{METHODOLOGY}

The algorithmic steps are:

a. Read gray scale image, $x$.

b. Generate a noisy image y by adding Gaussian noise.

c. Compute weighted average filter over noisy image y using a $3 \times 3$ weighted mask on $3 \mathrm{X} 3$ neigh hood within the image.

d. Repeat step c above to cover the entire image.

e. Calculate peak signal to noise ratio (PSNR) of $d$ above.

f. Investigate edges from noisy image y by computing difference between neighbouring pixels and compare with preselect threshold and store horizontal, vertical, left diagonal and right diagonal edges of an image in separate arrays.

g. Combiner algorithm scans the array $\{h 1\}$. If it encounters a non-zero element, it removes edge sample from the edge filter output and replaces the corresponding samples of the weighted average smoother output with the removed samples. This procedure is repeated for all non-zero values of $\{\mathrm{h} 1\}$. Similarly, the combiner scans the arrays $\{\mathrm{h} 2\},\{\mathrm{h} 3\}$ and $\{h 4\}$ in sequence. Finally it creates enhanced image with edges.

h. Calculate peak signal to noise ratio (PSNR) of enhanced image.

i. Compare PSNR of enhanced image with that of blurred image obtained in step $d$ above.

\section{RESULTS AND DISCUSSION}

The test images considered for this study are given in Figures 4.1 and 4.2. The results are presented in the order of input image, noisy image, weighted average filter output and proposed new filter output (enhanced image).

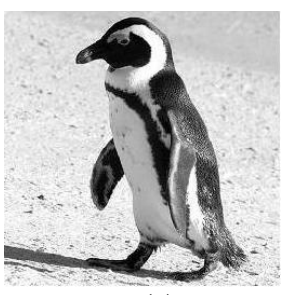

(a)

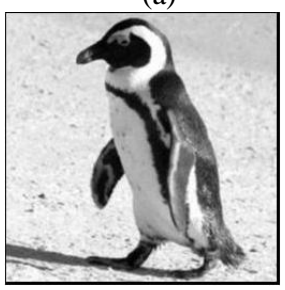

(c)

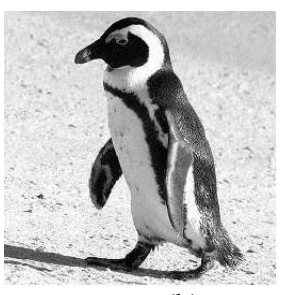

(b)

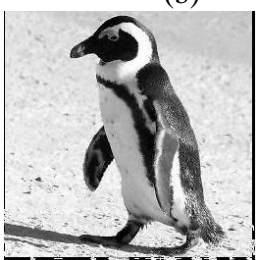

(d)
Fig4.1: (a) input image (pengiun.jpg) (b) noisy image $($ st.deviation $=5)(c)$ Weighted Average filter output image with $P S N R=14.81 \mathrm{db}(\mathrm{d})$ New filter output image with $P S N R=16.69 \mathrm{db}$ 


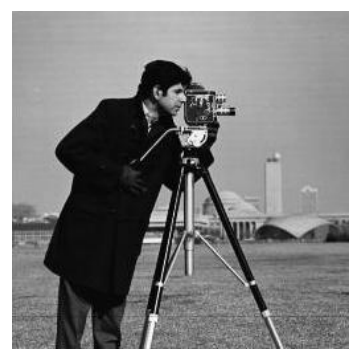

(a)

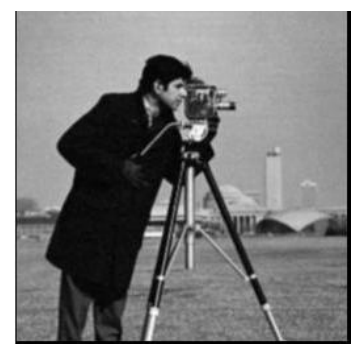

(c)

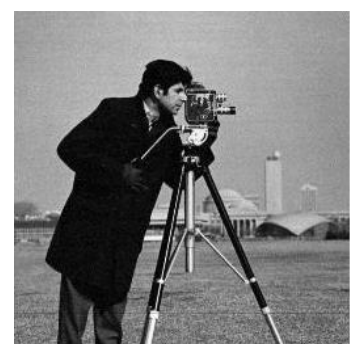

(b)

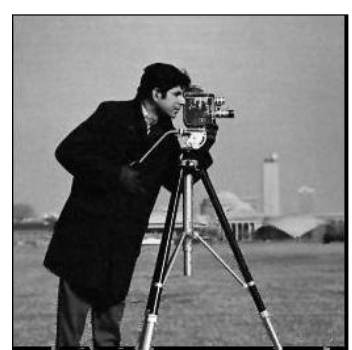

(d)
Fig4.2: (a) input image (cameraman.tif) (b) noisy image (st.deviation=5)(c) Weighted Average filter output image with $P S N R=17.97 \mathrm{db}(\mathrm{d})$ New filter output image with PSNR=20.

Table 4.1: Comparision of PSNR(db)for different testimag

\begin{tabular}{|c|c|c|c|c|c|}
\hline $\begin{array}{c}\text { Standard } \\
\text { deviation }\end{array}$ & 5 & 10 & 20 & 30 & 40 \\
\hline Test image & \multicolumn{5}{|c|}{ Penguin.jpg(256X256) } \\
\hline $\begin{array}{c}\text { Weighted } \\
\text { Average }\end{array}$ & 14.81 & 14.67 & 14.10 & 13.31 & 12.44 \\
\hline New Filter & 16.69 & 16.71 & 17.22 & 20.53 & 22.53 \\
& & & & & \\
\hline Test image & & & & & \\
\hline Weighted & 17.97 & 17.65 & 16.63 & 15.29 & 13.95 \\
\hline Average & & & & & \\
\hline New Filter & 20.26 & 19.92 & 19.51 & 22.24 & 24.72 \\
& & & & & \\
\hline
\end{tabular}

The test image used is the picture of cameraman.tif $(256 \times 256$ pixels, 8 bits/pixel) and pengiun.jpg (256x256 pixels, 8 bits/pixel). PSNR [3] is the logarithmic value of the ratio of size of the image and the mean square error (MSE) of the image.

$M S E=\frac{1}{M N} \sum_{i=1}^{M} \sum_{j=1}^{N} o(i, j)-y(i, j)$
$P S N R=10 \log _{10}\left(255^{2} / M S E\right)$

From the above Table, it may be seen that the image enhancement achieved using New Filter is superior to that obtained using the weighted average filter for all noise levels (standard deviation) considered. Weighted average filter, as can be seen, suppresses uniform noise satisfactorily; but it fails to preserve the edge information and therefore, the image is not pleasant to view. The New Filter attenuates uniform noise as effectively as the weighted average filter while preserving the image boundaries and fine details faithfully.

\section{CONCLUSIONS}

A simple and novel approach is developed to enhance the digital images by constructing the proposed New Filter. The new filter is constructed by combining a weighted average filter with an edge filter. The results obtained in terms of peak signal to noise ratio levels are encouraging. This algorithm may be implemented for real life applications.

\section{REFERENCES}

[1] Raman Maini and Himanshu Aggarwal, A Comprehensive Review of Image Enhancement Techniques, JOURNAL OF COMPUTING, VOLUME 2, ISSUE 3, MARCH 2010.

[2] Chandra Sekhar Panda, Prof. (Dr.) Srikanta Patnaik. "Filtering corrupted image and edge detection in restored gray scale image using derivative filters", International journal of image processing (IJIP), volume 3, issue 3, 2008.

[3] Ashish Verma1 and Bharti Sharma, Comparative Analysis in Medical Imaging, International Journal of Computer Applications, Volume 1 - No. 13, 2010.

[4] Gonzalez, R.C.; Woods, R.E. Digital Image Processing; Addison-Wesley, 2002.

[5] Pratt W. Digital Image Processing; John Wiley and Sons, 2001

[6] Jain, A. Fundamentals of Digital Image Processing; Prentic Hall, 1988.

[7] M.sridevi and P.jenopaul'Image enhancement using spatial filtering"Journal of Engineering and Applied Sciences, Year: 2011, Volume: 6 ,Issue: 2 ,Page No.: 180-184

[8] V.Saradhadevi , Dr.V.Sundaram," A Survey on Digital Image Enhancement Techniques" (IJCSIS) International Journal of Computer Science and Information Security, Vol. 8, No. 8, November 2010 